\title{
Reproduzierbarkeit der FEV 1 bei Kindern von 4 bis 6 Jahren mit intermittierendem Asthma bronchiale im beschwerdefreien sowie im Beschwerdeintervall*
}

\author{
The Repeatability of Forced Expiratory Manoeuvres in 4- to 6-Year-Old Children with Intermittent Bronchial \\ Asthma in Healthy and in Exacerbated Status
}

Autoren

Institute
M. Christmann, S. von Erffa, M. Rosewich, M. A. Rose, J. Schulze, S. Zielen

Allergologie, Pneumologie und Mukoviszidose, Zentrum für Kinder- und Jugendmedizin, J. W. Goethe-Universität Frankfurt am Main eingereicht 8.4 .2010 akzeptiert nach Revision 29. 6. 2010

\section{Bibliografie}

Dol http://dx.doi.org/ $10.1055 / \mathrm{s}-0030-1255625$

Online-Publikation: 23. 8. 2010

Pneumologie 2010; 64:

745-751 @ Georg Thieme

Verlag KG Stuttgart · New York ISSN 0934-8387

\section{Korrespondenzadresse}

Dr. med. Martin Christmann

Klinikum der Johann Wolfgang

Goethe-Universität

Zentrum für Kinder- und Jugendmedizin

Allergologie, Pneumologie und Mukoviszidose

Theodor-Stern-Kai 7

60590 Frankfurt am Main

martin.christmann@kgu.de

\section{Zusammenfassung \\ $\nabla$}

Einleitung: Die Frage nach reproduzierbaren exspiratorischen Manövern in der Lungenfunktionsmessung im Kindesalter ist von klinischem sowie wissenschaftlichem Interesse. Inwieweit Kinder mit intermittierendem frühkindlichem Asthma bronchiale im Alter von $\geq 4$ bis $<7$ Jahren sowohl im klinisch gesunden wie auch im exazerbierten Zustand reproduzierbare Lungenfunktionsmessungen durchführen können, wurde in der vorliegenden Arbeit analysiert.

Methode: Bei 64 Kindern mit frühkindlichem Asthma bronchiale im Alter $\geq 4$ bis $<7$ Jahren wurden jeweils im gesunden und exazerbierten Zustand Lungenfunktionsuntersuchungen durchgeführt. Die $\mathrm{FEV}_{1}$-Werte aller Messungen wurden nach den ATS/ERS-Leitlinien von 2007 hinsichtlich Reproduzierbarkeit untersucht.

Ergebnisse: Im gesunden Intervall zeigen nach ATS-Kriterien 74,6\% der Kinder mindestens 2 und 59,3\% 3 reproduzierbare Messungen. Im exazerbierten Intervall sind es $87,5 \%$ für mindestens 2 und $68,8 \%$ für 3 reproduzierbare Messungen. Es zeigen sich keine Unterschiede zwischen gesundem und exazerbiertem Zustand. Zwischen den einzelnen Altersgruppen zeigen sich keine signifikanten Unterschiede. Kinder der untersuchten Altersgruppe schaffen signifikant mehr reproduzierbare Messungen nach neuen Leitlinien, verglichen mit früheren Reproduzierbarkeitskriterien $(\mathrm{p}<0,0001)$.

Schlussfolgerung: Die ATS/ERS-Kriterien von 2007 stellen im Vergleich zu früheren Kriterien eine Vereinfachung für die Reproduzierbarkeit der exspiratorischen Manöver bei Kindern im Alter von 4-6 Jahren dar. Die Reproduzierbarkeit ist in dieser Altersgruppe im Rahmen von Exazerbationen nicht vermindert. Nach aktuellen ATS/ ERS-Kriterien können 74,6\% der Kinder reproduzierbar eine Lungenfunktion durchführen.

\section{Abstract \\ V}

Introduction: The question about the repeatability of forced expiratory manoeuvres in childhood lung function testing is of scientific and clinical interest. The following study investigated to what extent children $\geq 4$ to $<7$ years of age with intermittent bronchial asthma are able to produce reproducible lung function measurements on the one hand in the healthy status and on the other hand in an exacerbated status.

Method: 64 children at the age of $\geq 4$ to $<7$ years with intermittent preschool bronchial asthma performed lung function measurements in the healthy status and again in an exacerbated status. $\mathrm{FEV}_{1}$ values from the measurements were analysed according to ATS/ERS guidelines concerning repeatability.

Results: According to the new ATS/ERS guidelines $74.6 \%$ of the children could perform at least 2 , and $59.3 \%$ could perform 3 repeatable measurements in the healthy status. In the exacerbated status this was $87.5 \%$ and $68.8 \%$, respectively. There were no significant differences between the healthy and the exacerbated status and between the age groups. Compared to former repeatability criteria, children of this age group can perform significantly more reproducible measurements $(\mathrm{p}<0.0001)$.

Conclusion: The ATS/ERS guidelines from 2007 simplify the repeatability of forced expiratory manoeuvres in children at $\geq 4$ to $<7$ years of age compared to the former criteria. Repeatability is not reduced in the exacerbated status. $74.6 \%$ of children in this age group can produce repeatable lung function measurements.

\footnotetext{
* Teile der vorliegenden Arbeit wurden als Vortrag auf den Jahrestagungen der Gesellschaft für pädiatrische Pneumologie 2010 in Berlin sowie der Deutschen Gesellschaft für Pneumologie und Beatmungsmedizin 2010 in Hannover vorgestellt.
} 


\section{Einleitung}

Die Lungenfunktionsuntersuchung und insbesondere die Spirometrie gehören zu den etablierten diagnostischen Maßnahmen in der pneumologischen Praxis. Neben einer ausführlichen Anamnese, insbesondere auch der Familienanamnese und der körperlichen Untersuchung, ist die Spirometrie für die Untersuchung von obstruktiven Atemwegserkankungen wie dem Asthma bronchiale essenziell [1]. Dies gilt auch für Kinder, bei denen die sogenannten „Wheezing disorders“ außerordentlich häufig auftreten und die Abgrenzung zum Asthma bronchiale schwierig ist [1,2]. Lungenfunktionsuntersuchungen werden bereits ab dem frühen Kindesalter durchgeführt [3-6] und bedürfen neben erfahrenem und besonders in der Durchführung von Lungenfunktionsuntersuchungen bei Kindern geschultem Personal auch computergesteuerte Animationsprogramme [7]. Aufgrund des jungen Alters und der häufig fehlenden Compliance bei der Durchführung der Untersuchung beschäftigten sich sowohl die gemeinsame Veröffentlichung der American Thoracic Society (ATS) und European Respiratory Society (ERS) und die Gesellschaft für pädiatrische Pneumologie (GPP) mit der Fragestellung, inwieweit Kinder im Vorschulalter bereits verlässliche, reproduzierbare Lungenfunktionen durchführen können [3,4,8,9]. Diese Frage ist nicht nur von rein wissenschaftlichem Interesse, sondern gerade in der täglichen Praxis ein wichtiger Baustein, um die Diagnose Asthma bronchiale korrekt zu stellen.

Die Leitlinien zur Reproduzierbarkeit der Lungenfunktion galten bis vor kurzem hauptsächlich für Erwachsene [10,11]. Diese sollten allerdings für Kinder im Vorschulalter aufgrund der geringeren Lungenvolumina und der kürzeren Atemmanöver nicht angewendet werden [3]. Aus diesem Grund haben ATS und ERS im Jahr 2007 aktualisierte Leitlinien vorgestellt, die neue Kriterien zum einen für die Akzeptanz einer Messung sowie die Reproduzierbarkeit einer Messung darstellen [3]. Von Seiten der ATS/ERS gelten in den aktualisierten Leitlinien die forcierten exspiratorischen Manöver als reproduzierbar, wenn zwei akzeptable Kurven vorliegen, die weniger als $0,1 \mathrm{~L}$ bzw. $10 \%$ vom jeweils höchsten Wert (FVC, FEV 1 ) abweichen.

Besonders wichtig erscheint allerdings die Tatsache, dass eine gute Reproduzierbarkeit nicht nur im gesunden, beschwerdefreien Intervall wie zum Beispiel bei elektiven Untersuchungen im Rahmen einer wissenschaftlichen Fragestellung gegeben ist, sondern dass die Spirometrie auch während einer akuten Episode mit Hustenbeschwerden verlässliche, wiederholbare Aussagen über eine evtl. vorliegende Obstruktion geben kann. Die tägliche Praxis lässt vermuten, dass Kinder mit diagnostiziertem Asthma bronchiale bei Exazerbation weniger wiederholbare Lungenfunktionsmessungen produzieren können. Zu dieser Fragestellung liegen jedoch bisher keine Daten vor, da bisher alle Untersuchungen ausschließlich bei gesunden Kindern nach entsprechendem Training durchgeführt wurden. Da dies nicht der täglichen Praxis entspricht, wurde im Rahmen einer klinischen Untersuchung während und nach erfolgreicher Behandlung einer Exazerbation die Reproduzierbarkeit der forcierten exspiratorischen Manöver der Lungenfunktion bei jungen Kindern mit Asthma im Alter von $\geq 4$ bis $<7$ Jahren untersucht.

\section{Methode \\ $\nabla$}

Die vorliegende Untersuchung wurde an der Universitätskinderklinik Frankfurt am Main als eine Substudie bei Kindern mit frühkindlichem Asthma bronchiale durchgeführt. Ziel der Hauptstudie war die Charakterisierung von Responderprofilen auf eine anti-inflammatorische Kurzzeit-Therapie im Kindesalter. Teile dieser Studie wurden bereits Anfang 2010 veröffentlicht [12]. Eingeschlossen in die Untersuchung wurden Kinder von $\geq 4$ bis $<7$ Jahren mit der Diagnose eines intermittierenden frühkindlichen Asthma bronchiale. Alle Kinder waren bei Einschluss in die Studie beschwerdefrei. Ausschlusskriterien waren medikamentöse Dauertherapie eines Asthma bronchiale, orale Steroide, schwere andere Begleiterkrankungen (z.B. Mukoviszidose, maligne Erkrankungen) sowie die Teilnahme an einer anderen klinischen Studie. Von insgesamt 142 aufgenommenen Kindern im Alter von $\geq 4-<7$ Jahren wurden 64 Kinder (45,1\%) im Rahmen einer Exazerbation ihres Asthma bronchiale, definiert als erhöhter Salbutamolbedarf ( $\geq 3$ Hübe pro Woche oder $\geq 5$ Hübe in zwei Wochen), entsprechend den festgelegten Studienkriterien in einen der beiden Therapiearme, entweder Fluticasone oder Montelukast, randomisiert. Nach 6 Wochen anti-inflammatorischer Therapie mit einem der beiden Medikamente erfolgte die Therapieevaluation.

Als eine wichtige Substudie der genannten Untersuchung wurde die Reproduzierbarkeit der forcierten exspiratorischen Manöver der Lungenfunktion der 64 randomisierten Kinder zum einen bei Exazerbation sowie zum anderen im beschwerdefreien Intervall nach 6 Wochen anti-inflammatorischer Therapie untersucht. Es wurden zu beiden Zeitpunkten jeweils drei Wiederholungen der Lungenfunktionsmessung durchgeführt.

Zu beiden Zeitpunkten wurden zunächst die Basisparameter erhoben (Alter, Größe, Gewicht, BMI, Puls, Sauerstoffsättigung) und jeweils die Spirometrie durchgeführt. Bei Exazerbation schloss sich an die Spirometrie eine Bronchospasmolyse mit 2 Hüben Salbutamol je $100 \mu$ ü über den Aerochamber ${ }^{\circledR}$ an. Bei Besserung der $\mathrm{FEV}_{1}(\%)$-Werte nach Salbutamol-Inhalation um mehr als 5\% vom höchsten Ausgangswert wurden 2 weitere Hübe Salbutamol je $100 \mu g$ gegeben, bis zu einem Maximum von 6 Hüben (kumulierte Dosis von $600 \mu \mathrm{g}$ Salbutamol). Die Untersuchungen wurden jeweils von in der Durchführung von Kinder-Lungenfunktionsuntersuchungen erfahrenem Personal betreut.

Die Spirometrie wurde mit Hilfe des computergestützen Spirometers Jäger Masterscreen IOS der Firma VIASYS Healthcare $\mathrm{GmbH}$, Höchberg, durchgeführt. Bei jeder Messung war mindestens ein Elternteil anwesend. Die Untersuchungen begannen mit einer ausführlichen Instruktion der Probanden, der Untersuchungsgang wurde dabei beschrieben und demonstriert. Um die Mitarbeit zu verbessern, wurden die Messungen mit Computerspielen animiert. Es wurde darauf geachtet, dass sich die Probanden während der Messungen in aufrechter Sitzposition mit leicht rekliniertem Kopf befanden. Die Nase war während aller Messungen mit einem Nasenclip verschlossen. Bei jeder Spirometrie erfolgten jeweils 3 Wiederholungen und es wurden folgende Messwerte für die Auswertung erhoben: VC (1), VC (\%), FVC (1), FVC (\%), FEV 1 (1), FEV 1 (\%), FEV ${ }_{0,5}$ (1) Tiffenau-Index, VBE, VBE\% FVC. Die Werte von FVC und $\mathrm{FEV}_{1}$ sowie ggf. $\mathrm{FEV}_{0,5}$ einer jeden Messung wurden für die Bestimmung der Reproduzierbarkeit ausgewertet. Als Start-of-Test Kriterien wurden entsprechend der genannten Leitlinien das „back-extrapolated Volume“ (VBE) sowie das Verhältnis von VBE zu FVC beurteilt (VBE/FVC). Zur Beurteilung der End-of-Test Kriterien diente die Begutachtung jedes einzelnen Kurvenverlaufs durch den Auswerter. 


\begin{tabular}{|llllll|}
\hline & & Gesamt & 4-Jährige & 5-Jährige & 6-Jährige \\
\hline Alter (J) & MW (SD) & $5,67(0,78)$ & & & \\
\hline Anzahl & $\mathrm{n}(\%)$ & $64(100)$ & $21(32.8)$ & $25(39,1)$ & $18(28,2)$ \\
\hline männlich & $\mathrm{n}(\%)$ & $41(0,64)$ & $13(61,9)$ & $17(68,0)$ & $11(61,1)$ \\
\hline Größe (cm)* & $\mathrm{MW}(\mathrm{SD})$ & $115,0(7,8)$ & $108,8(4,9)$ & $114,2(5,6)$ & $123,6(5,2)$ \\
\hline Gewicht (kg)* & $\mathrm{MW}(\mathrm{SD})$ & $21,5(5,2)$ & $18,4(2,1)$ & $20,7(3,4)$ & $26,3(6,2)$ \\
\hline BMI* $^{*}$ & $\mathrm{MW}(\mathrm{SD})$ & $16,1(2,6)$ & $15,5(1,1)$ & $15,9(2,0)$ & $17,2(3,9)$ \\
\hline elterliches Rauchen & $\mathrm{n}(\%)$ & $25(39,1)$ & $8(38,1)$ & $9(36,0)$ & $8(44,4)$ \\
\hline
\end{tabular}

Tab. 1 Basisparameter der untersuchten Kinder.

* kennzeichnet die signifikanten Unterschiede der einzelnen Gruppen.

Tab. 2 Lungenfunktionswerte der untersuchten Kindergruppen.

\begin{tabular}{|c|c|c|c|c|c|c|c|c|c|}
\hline & & \multicolumn{2}{|c|}{ Gesamt } & \multicolumn{2}{|c|}{ 4-Jährige } & \multicolumn{2}{|c|}{ 5-jährige } & \multicolumn{2}{|c|}{ 6-Jährige } \\
\hline & & $\mathrm{E}$ & G & E & G & E & G & E & G \\
\hline FVC & (I) & 1,28 & 1,41 & 1,05 & 1,07 & 1,21 & 1,30 & 1,59 & 1,77 \\
\hline $\mathrm{FEV}_{1}$ & $(\%)$ & 91,8 & 105,3 & 90,1 & 105,5 & 89,7 & 102,7 & 96,9 & 109,1 \\
\hline Tiffeneau & & 80,4 & 85,5 & 79,6 & 88,4 & 79,8 & 83,4 & 82,2 & 84,6 \\
\hline $\begin{array}{l}\text { Bronchospasmolyse } \\
\geq 15 \% \mathrm{FEV}_{1}\end{array}$ & $\mathrm{n}(\%)$ & \multicolumn{2}{|c|}{$14(21,9)$} & \multicolumn{2}{|c|}{$4(19,0)$} & \multicolumn{2}{|c|}{$6(24,0)$} & \multicolumn{2}{|c|}{$4(22,2)$} \\
\hline
\end{tabular}

E: Exazerbation; G: Gesund/Beschwerdefrei.

Eine Lungenfunktionsmessung gilt nach ATS/ERS im jungen Kindesalter als reproduzierbar, wenn zwei akzeptable Kurven vorliegen, deren Werte für FVC bzw. FEV 1 um weniger als $0,1 \mathrm{~L}$ bzw. $10 \%$ vom höchsten Wert abweichen. Entsprechend dieser Definition wurden die einzelnen Parameter zunächst bei Exazerbation und zum Vergleich im beschwerdefreien Intervall bestimmt. Zusätzlich wurde ein Vergleich zwischen neuen Reproduzierbarkeitskriterien nach ATS/ERS wie oben beschrieben und den zuvor geltenden Kriterien der ATS/ERS durchgeführt, welche noch eine Abweichung von $\leq 5 \%$ vom jeweils höchsten Wert für $\mathrm{FEV}_{1}$ und FVC forderten $[11,13]$.

Die statistische Auswertung wurde durch Bestimmung der absoluten sowie prozentualen Zahlen, der Mittelwerte, Mediane und Standardabweichungen sowie mittels T-Test für verbundene und unverbundene Stichproben durchgeführt.

\section{Ergebnisse}

An der Untersuchung nahmen insgesamt $\mathrm{n}=64$ Kinder teil. $\mathrm{N}=41$ (64,1\%) davon waren männlichen, $\mathrm{n}=23$ (35,9\%) weiblichen Geschlechts. Im Mittel waren die Kinder bei der Untersuchung 5,7 Jahre alt. Die Basisparameter des Patientenkollektivs sind in Tab. 1 dargestellt.

Zur Evaluation der Reproduzierbarkeiten in den jeweiligen Altersgruppen wurden die Kinder nach Alter in folgende Gruppen, eingeteilt: Gruppe 1: 4-< 5 Jahre; Gruppe 2: 5-<6 Jahre; Gruppe 3: $6-<7$ Jahre. Hinsichtlich Größe, Gewicht und BMI unterschieden sich die drei Gruppen signifikant voneinander ( $\bullet$ Tab. 1).

Die einzelnen Lungenfunktionswerte der Patientengruppen zeigt - Tab.2, aufgeteilt in Werte im Rahmen der Exazerbation und während des gesunden Intervalls nach Therapie.

Laut ATS/ERS-Kriterien werden im Vorschulalter entsprechend der neuen Leitlinien eine VBE von $80 \mathrm{ml}$ bzw. 12,5\% der FVC für ein adäquates „Start-of-Test“-Kriterium gefordert. Alle in unsere Untersuchung aufgenommenen Kinder erreichten diese Forderung ( $\mathrm{n}=64,100 \%)$.

Zunächst analysierten wir die Zeiten der forcierten Exspiration (FET) des Patientenkollektivs ( $\bullet$ Abb. 1).

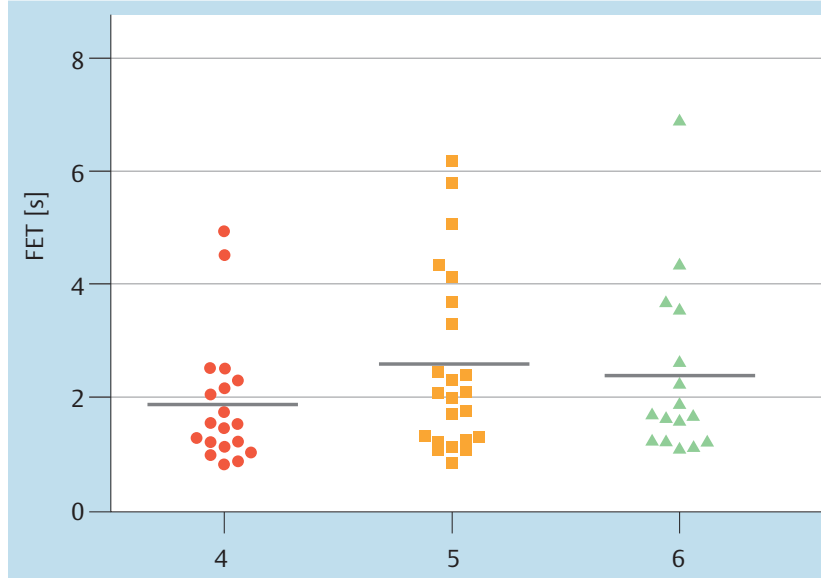

a Alter (Jahre)

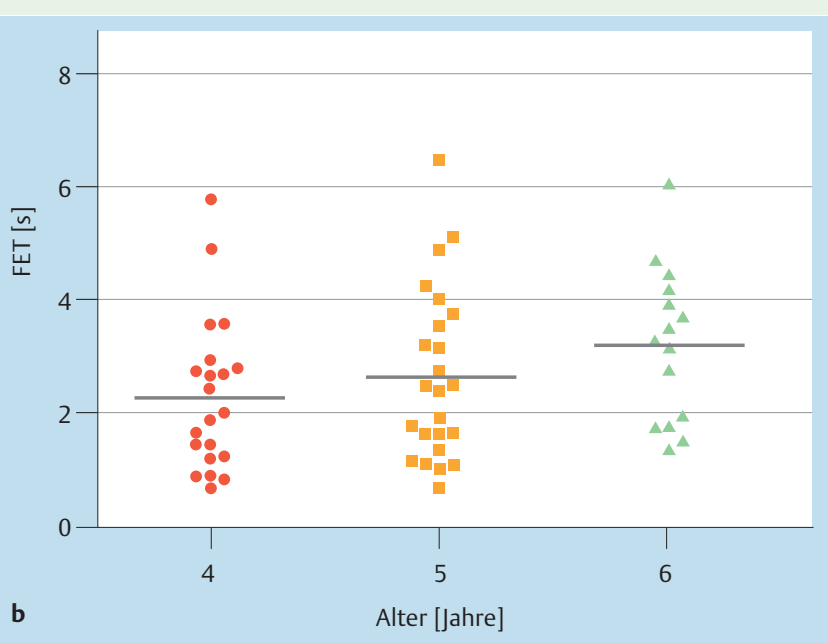

Abb. 1 FET-Werte der einzelnen Altersgruppen (a) im beschwerdefreien Intervall und (b) bei Exazerbation. 


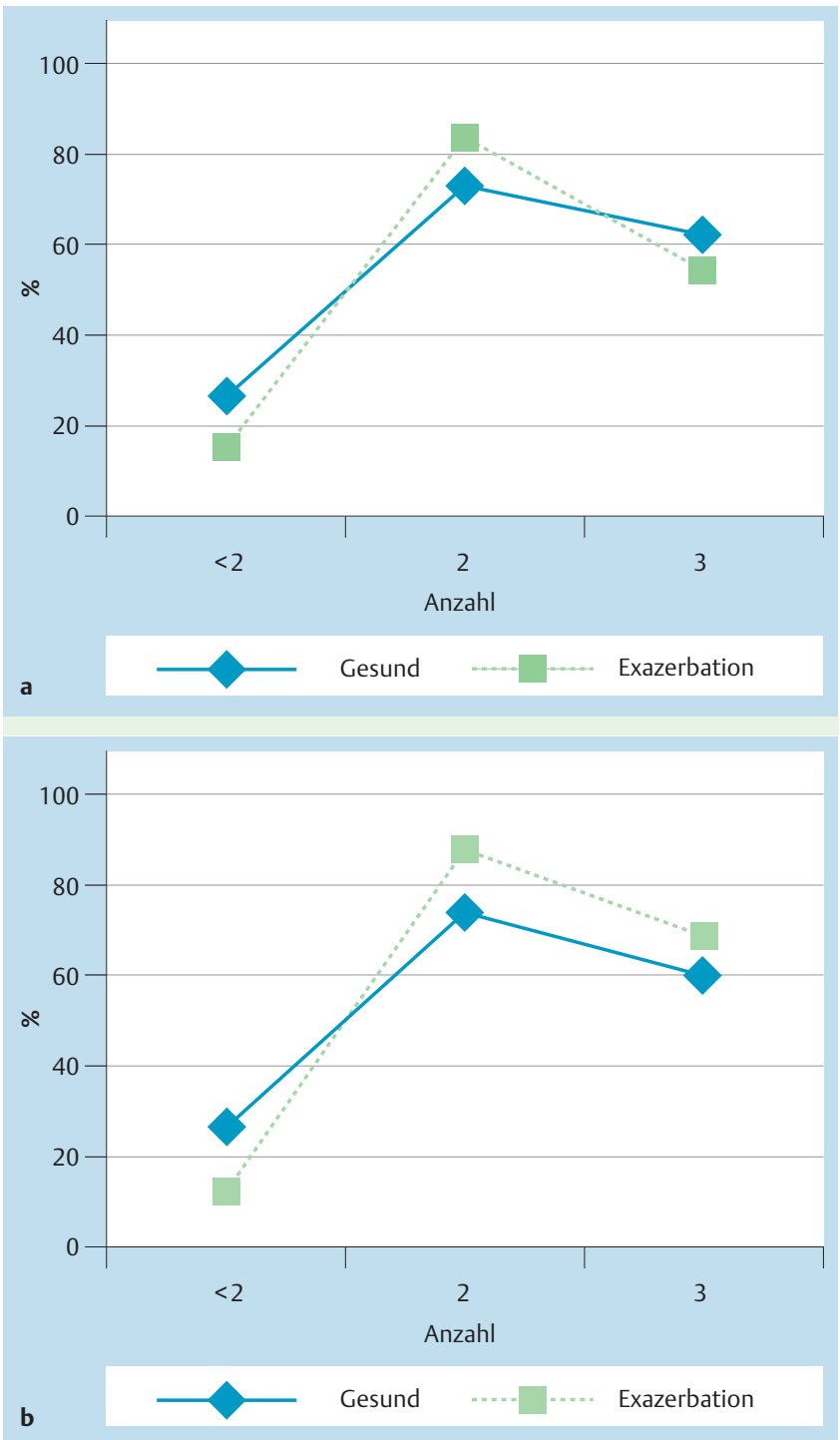

Abb. 2 Prozentuale Darstellung der Anzahl reproduzierbarer Messungen nach neuen ATS/ERS-Kriterien in gesundem und exazerbiertem Intervall für (a) FVC (\%) und (b) FEV 1 (\%).
Bei Exazerbation zeigte sich die FET im Vergleich zum beschwerdefreien Intervall verlängert (2,7 vs. 2,3 Sekunden), ohne allerdings statistische Signifikanz zu erreichen $(p=0,066)$. Auch in den einzelnen Altersgruppen zeigte sich eine verlängerte FET bei Exazerbation, ein 5\%-Signifikanzniveau zeigte sich lediglich bei der Gruppe der 6-jährigen Kinder (3,2 vs. 2,4 Sekunden; $p$ =0,02). Eine FET von < 1 Sekunde zeigte sich im Beschwerdeintervall bei $n=5$ Kindern (4-jährige: $n=4$; 5 -jährige: $n=1$ ), im beschwerdefreien Intervall bei $n=4$ Kindern (4-jährige: $n=3$; 5 -jährige: $\mathrm{n}=1)$.

Die Auswertung der reproduzierbaren Lungenfunktionen erfolgte anhand der forcierten exspiratorischen Manöver ( $\mathrm{FEV}_{1}$ sowie FVC). Dabei untersuchten wir die Lungenfunktionen auf Reproduzierbarkeit jeweils im exazerbierten sowie im gesunden Intervall.

Bezogen auf die $\mathrm{FEV}_{1}$-Werte schafften im gesunden Intervall 74,6\% aller Kinder mindestens 2 reproduzierbare Lungenfunktionen, 59,3\% schafften 3 reproduzierbare Messungen. Im kranken Intervall schafften 87,5\% mindestens 2 und 68,7\% 3 reproduzierbare Lungenfunktionen. Zwischen gesundem und krankem Stadium zeigten sich keine signifikanten Veränderungen ( $p=0,22$ ). Bezüglich der FVC-Werte zeigte sich, dass 73,3\% aller Patienten im gesunden Intervall mindestens 2 reproduzierbare Lungenfunktionen produzieren können. Drei reproduzierbare Werte schafften $61,7 \%$. Im kranken Intervall waren es $83,6 \%$ für mindestens 2 und 54,1\% für 3 reproduzierbare Messungen. Zwischen gesundem und krankem Stadium zeigte sich hier ebenfalls kein signifikanter Unterschied ( $p=0,36)$. Entgegen unserer initialen Annahme konnten im Rahmen der Exazerbation, gerade bei den $\mathrm{FEV}_{1}$-Werten, jeweils mehr reproduzierbare Ergebnisse erzielt werden als im beschwerdefreien Intervall ( $\bullet$ Abb. 2).

Eine detaillierte Aufstellung der Reproduzierbarkeiten innerhalb der einzelnen Altersgruppen sowie einen Vergleich zu früheren Reproduzierbarkeitskriterien zeigt $\bullet$ Tab. 3.

Für Kinder mit einer FET $<1$ Sekunde analysierten wir die $\mathrm{FEV}_{0,5^{-}}$ Werte. Hierzu liegen in dem von uns verwendeten Messsystem lediglich die absoluten $\mathrm{FEV}_{0,5}$-Werte vor. Es zeigte sich, dass von den 5 Patienten mit einer FET< 1 Sekunde bei Exazerbation 4 mindestens 2 reproduzierbare Messungen produzieren konnten (80\%), einer schaffte 3 reproduzierbare Messungen (20\%). Im gesunden Intervall zeigten alle Patienten mindestens 2 reprodu-

\begin{tabular}{|c|c|c|c|c|c|}
\hline & \multirow[b]{2}{*}{$\begin{array}{l}\text { Anzahl repr. } \\
\text { Messungen } \\
\left(\mathrm{FEV}_{1}\right)\end{array}$} & \multicolumn{2}{|c|}{ Gesundes Intervall } & \multicolumn{2}{|c|}{ Exazerbation } \\
\hline & & Neu & Alt & Neu & Alt \\
\hline \multirow[t]{3}{*}{4 Jahre } & 0 & $31,6 \%$ & $52,6 \%$ & $14,3 \%$ & $47,6 \%$ \\
\hline & 2 & $68,4 \%$ & $47,4 \%$ & $85,7 \%$ & $52,4 \%$ \\
\hline & 3 & $47,4 \%$ & $42,1 \%$ & $61,9 \%$ & $28,6 \%$ \\
\hline \multirow[t]{3}{*}{5 Jahre } & 0 & $20,8 \%$ & $25,0 \%$ & $8,0 \%$ & $32,0 \%$ \\
\hline & 2 & $79,2 \%$ & $75,0 \%$ & $92,0 \%$ & $68,0 \%$ \\
\hline & 3 & $66,7 \%$ & $37,5 \%$ & $68,0 \%$ & $44,0 \%$ \\
\hline \multirow[t]{3}{*}{6 Jahre } & 0 & $25,0 \%$ & $50,0 \%$ & $16,6 \%$ & $33,3 \%$ \\
\hline & 2 & $75,0 \%$ & $50,0 \%$ & $83,3 \%$ & $66,7 \%$ \\
\hline & 3 & $62,5 \%$ & $43,7 \%$ & $77,8 \%$ & $38,9 \%$ \\
\hline
\end{tabular}

Tab. 3 Reproduzierbare $F E V_{1}$ Werte bei Kindern $\geq 4-<7$ Jahre im gesunden und kranken Intervall, aufgeteilt auf die neuen (ATS/ERS 2007) und alten Reproduzierbarkeitskriterien. 


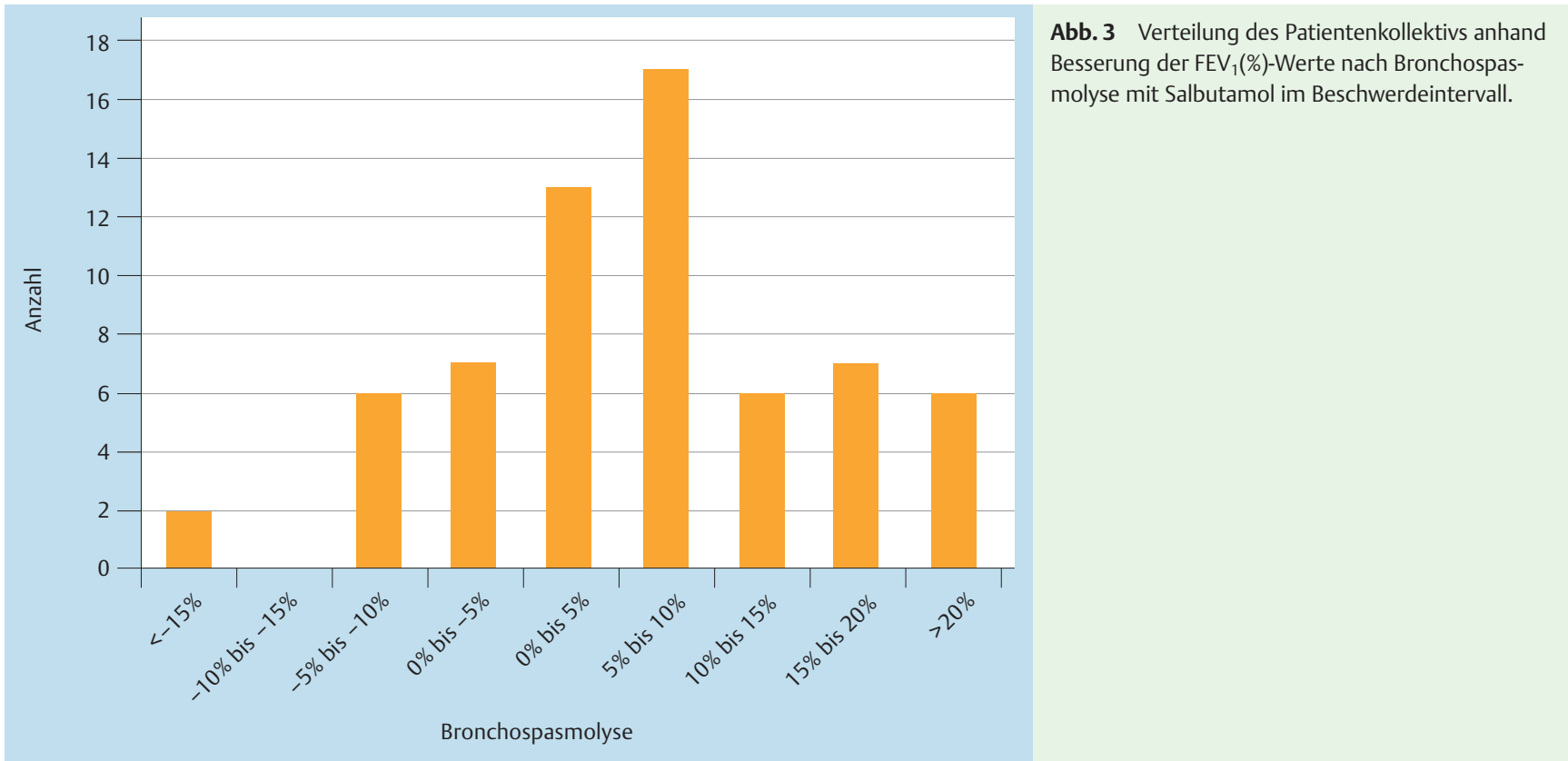

zierbare Messungen ( $n=4,100 \%)$, wobei erneut ein Patient 3 Messungen produzierte, welche die Reproduzierbarkeitskriterien erfüllten (25\%).

Zur Analyse, warum im kranken Intervall mehr reproduzierbare Messungen durchgeführt wurden, analysierten wir die Patienten, die bei Exazerbation eine positive Bronchospasmolyse mit maximaler Besserung der $\operatorname{FEV}_{1}(\%)$ nach Salbutamolinhalation um mehr als $15 \%$ aufwiesen, also diejenigen Patienten mit tatsächlicher Obstruktion. In $\bullet$ Abb. 3 und $\bullet$ Tab. 2 ist die Verteilung des Patientenkollektivs nach Besserung der $\mathrm{FEV}_{1}(\%)$-Werte nach Bronchospasmolyse im Exazerbationsstatus dargestellt.

- Abb. 4 zeigt exemplarisch Fluss-Volumen-Kurven vor und nach Bronchospasmolyse mit Salbutamol.

Eine Analyse der Patienten mit einer maximalen Bronchospasmolyse von $\geq 15 \%$ Anstieg von $\mathrm{FEV}_{1}(\%)$ gegenüber den Patienten ohne Bronchospasmolyse zeigte, dass im Exazerbationsstadium jeweils mehr reproduzierbare Ergebnisse durch Patienten mit positiver Bronchospasmolyse erzielt wurden als durch Patienten ohne positive Bronchospasmolyse ( Tab.4). Dieser Trend war allerdings nicht statistisch signifikant $(\mathrm{p}=0,48)$.

Verglichen mit früheren Kriterien $[11,13]$, in denen eine maximale Abweichung von 5\% vom jeweils höchsten Ausgangswert für eine reproduzierbare Messung gefordert wurde, zeigten sich sowohl im gesunden als auch im kranken Intervall nach neuen ATS/ERS-Kriterien in der Gesamtgruppe sowie in den altersunterteilten Gruppen signifikant mehr reproduzierbare Messungen (jeweils $\mathrm{p}<0,0001$, vgl. für die $\mathrm{FEV}_{1}$-Werte $\bullet$ Tab. 3).

\section{Diskussion}

Die vorliegende Untersuchung beschäftigt sich mit der Reproduzierbarkeit und damit verbunden auch der Wertigkeit der Lungenfunktionsmessungen bei Kindern im Alter von 4-7 Jahren. Es wird verglichen, ob im Rahmen einer Exazerbation eines frühkindlichen Asthma bronchiale im Vergleich zum beschwerdefreien Intervall die Reproduzierbarkeit herabgesetzt ist.

Zunächst konnten wir darstellen, dass die Akzeptanz einer Lungenfunktionsmessung in dieser Altersgruppe groß ist. Alle unse- re Patienten erreichten die geforderten „Start-of-test“-Kriterien mit einer VBE $<80 \mathrm{ml}$ bzw. einer VBE/FVC-Ratio von $<12,5 \%$. Bereits Loeb et al. [8] konnten zeigen, dass nahezu alle Kinder von 4-6 Jahren (97,0\%) dieses Kriterium erreichen. Da im Vorschulalter das „End-of-Test“-Kriterium nicht anhand eines Wertes definiert wird, liegt hier die Bewertung beim jeweiligen Auswerter. Bei Betrachtung der FET-Werte unseres Patientenkollektivs fällt auf, dass nahezu alle Patienten (92,2\% bzw. 93,8\%) eine forcierte Exspirationszeit von mindestens einer Sekunde haben. Wie zu erwarten war, zeigten sich in der Gruppe der 4-jährigen Kinder selten kürzere Exspirationszeiten, wobei auch hier FET-Werte $>1$ Sekunde von $81,0 \%$ bzw. 85,7\% der Kinder erreicht wurden ( $\bullet$ Abb. 1). Aufgrund dieser Daten lässt sich ableiten, dass ein $\mathrm{FEV}_{1}$-Wert auch in dieser Altersgruppe meist aussagekräftig ist. Dennoch gilt zu beachten, dass bei Kindern mit FET-Zeiten $<1$ Sekunde $\mathrm{FEV}_{0,75}$ bzW. $\mathrm{FEV}_{0,5}$ zur Bewertung herangezogen werden sollten. In diesem Fall müssen je nach Gerätehersteller die absoluten $\mathrm{FEV}_{0,5}$-Werte betrachtet werden.

Des Weiteren zeigen unsere Untersuchungen, dass Kinder von $\geq 4$ bis $<7$ Jahren sowohl im beschwerdefreien als auch im Beschwerdeintervall in 74,6\% bzw. 87,5\% mindestens 2 Messungen produzieren können, welche nach den adaptierten ATS/ERS-Kriterien von 2007 als reproduzierbar gelten. Die hier genannten Werte gelten für die $\mathrm{FEV}_{1}$-Werte, für die FVC waren sie nahezu identisch. Unsere Ergebnisse der Analyse der $\mathrm{FEV}_{0,5}$ bei Kindern mit FET $<1$ Sekunde zeigen, dass die Reproduzierbarkeit dieser Messungen sehr ähnlich denjenigen für die $\mathrm{FEV}_{1}$-Messungen in der Gesamtgruppe sind und somit ebenfalls zur Reproduzierbarkeitsbestimmung herangezogen werden können. Unsere Zahlen liegen dabei unter den Werten von Aurora et al. [14] (>90\%) sowie Nève et al. [5] (> 85\%), die Vorschulkinder untersuchten, und über den Werten von Crenesse et al. [15]. Insbesondere bei Aurora et al. wurde allerdings die $\mathrm{FEV}_{0,75}$ untersucht, im Gegensatz zu unserer Untersuchung. Zudem wurde in den genannten Arbeiten nicht zwischen beschwerdefreien und exazerbierten jungen Asthmatikern unterschieden.

Interessanterweise zeigten sich in unserer Untersuchung keine signifikanten Unterschiede zwischen den einzelnen Altersgruppen. Größe, Gewicht und BMI stellten keinen Einflussfaktor dar. 


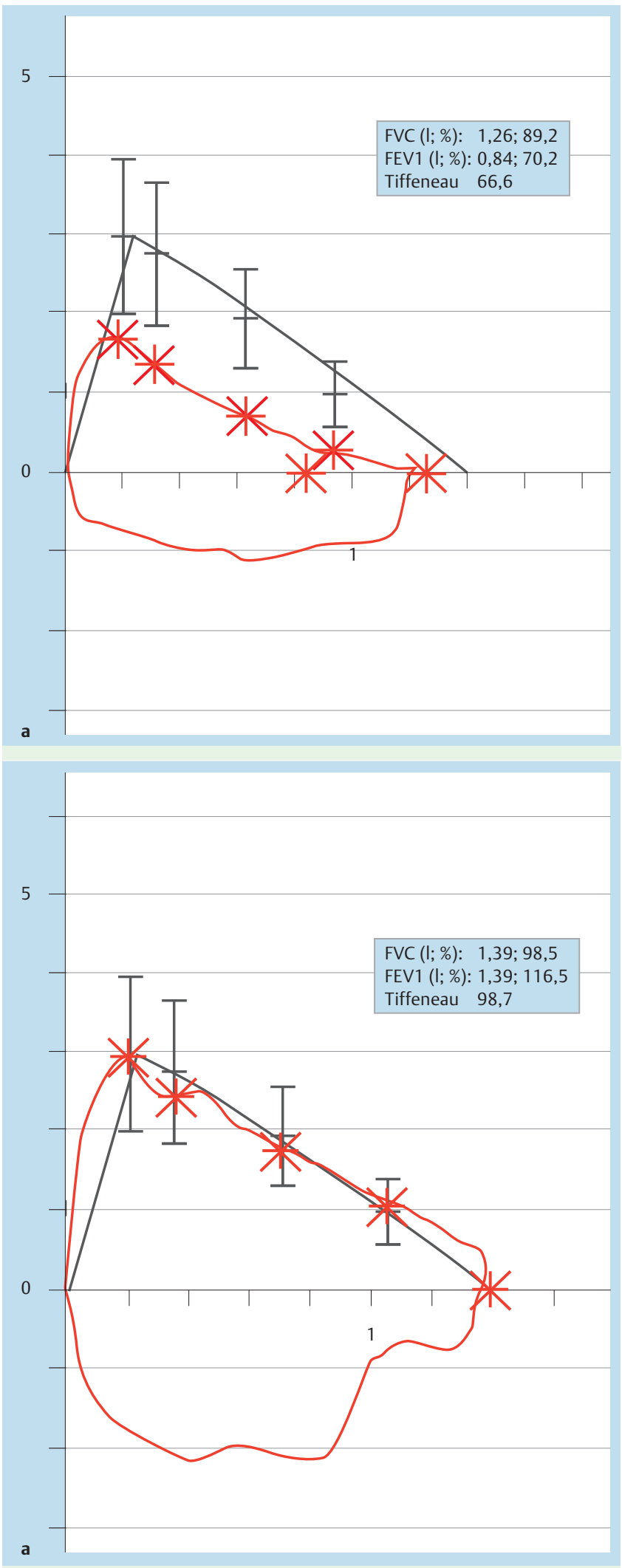

Abb. 4 Darstellung Fluss-Volumen-Kurve (a) vor Bronchospasmolyse und (b) nach Bronchospasmolyse.
Tab. 4 Anteil reproduzierbarer $\mathrm{FEV}_{1}(\%)-$ Werte bei Patienten mit und ohne signifikante Verbesserung von $\mathrm{FEV}_{1}$ nach Bronchospasmolyse mit Salbutamol bei Exazerbation.

\begin{tabular}{lll}
$\begin{array}{l}\text { Anzahl } \\
\text { reproduzierbare } \\
\text { Messungen }\end{array}$ & $\begin{array}{l}\text { Patienten mit } \\
\text { Bronchospasmolyse } \\
>\mathbf{1 5 \%} \text { FEV }_{\mathbf{1}}\end{array}$ & $\begin{array}{l}\text { Patienten ohne } \\
\text { Bronchospasmolyse }\end{array}$ \\
$\mathbf{< 2} \%$ FEV $_{\mathbf{1}}$ \\
\hline 2 & $7,1 \%$ & $13,3 \%$ \\
\hline 3 & $92,8 \%$ & $86,7 \%$ \\
\hline
\end{tabular}

Damit stimmen die Ergebnisse mit den Untersuchungen von Nève et al. [5] überein, die auch keinen Altersunterschied finden konnten. Andere Untersuchungen von Arets et al. [6] mit deutlich mehr Patienten konnten allerdings einen Alterseffekt zeigen. In unserer Gruppe der 6- bis 7-jährigen Patienten lag der Anteil der reproduzierbaren Messwerte (75,0\% im gesunden und 83,3\% im exazerbierten Intervall) deutlich unter denen der Schulkinder von Arets et al. Diese Autoren konnten zeigen, dass ältere Schulkinder in 85\% der untersuchten Kinder reproduzierbare Messungen nach den damals geltenden Richtlinien mit einer Abweichung von $\leq 5 \%$ vom jeweils höchsten Wert erzielten [11,13]. Nur im Beschwerdeintervall kommen unsere Zahlen an diese Ergebnisse heran, trotz neuem Bewertungsmaßstab mit Abweichung von $\leq 10 \%$ vom jeweils höchsten Wert. Auch andere Autoren beschreiben, dass die Werte von Arets et al. nicht erreicht werden $[4,14]$. In beiden Arbeiten wurden wie bei uns jüngere Kinder als bei Arets et al. untersucht. Der Unterschied erklärt sich somit vermutlich doch durch einen altersabhängigen Effekt (5,7 Jahre in unserer Untersuchung vs. 12,1 Jahre bei Arets et al.). Besonders wichtig für die Spirometrie bei kleinen Kindern ist das Umfeld, in dem die Untersuchungen durchgeführt werden, auch wenn hierzu bislang keine objektiven Studien zum Vergleich der Ergebnisse zwischen kinderadaptierten und erwachsenenorientierten Ambulanzen vorliegen. In unserer Untersuchung trugen alle Kinder bei den Messungen einen Nasenclip, auch wenn in Studien mit Kindern, welche allerdings älter waren als in unserer Untersuchung (5,7 vs. 11,4 Jahre), bislang kein signifikanter Messunterschied zwischen Messungen mit und ohne Nasenclip gefunden wurde [16].

Insbesondere computeranimierte Geräte können das Kind spielerisch an die verschiedenen Atemmanöver heranführen [7], auch wenn nicht alle Untersuchungen einen Vorteil solcher Programme zeigen konnten [16]. Unserer Erfahrung nach sollten diese Animationsprogramme entgegen der aktuell häufig verwendeten Animationen verschiedene Phasen beinhalten: 1. Phase: Hinführen zur maximalen Inspiration, beispielsweise als ein sich spannender Bogen eines Pfeil-und-Bogen-Schützen. 2. Phase: Maximale Exspiration, wie beispielsweise beim Kerzenausblasen. 3. Phase: Möglichst lange Exspirationsphase, wie beim langsamen Rollen eines Kegels. In geeignetem Umfeld lässt sich eine verwertbare Messung jedoch auch ohne Animationsprogramme durchführen, hierzu bedarf es unserer Ansicht nach allerdings mehr Erklärungsgeschick des Untersuchers, der am besten die Atemmanöver der Messung in einzelnen Phasen mit den Kindern vorher übt.

Interessanterweise war die Reproduzierbarkeit der Messungen im Rahmen der Exazerbation etwas besser als im gesunden, beschwerdefreien Intervall. Dieser überraschende Befund ist schwer zu erklären und veranlasste uns zu prüfen, ob sich Patienten mit einer signifikanten Bronchospasmolyse $(\geq 15 \%$ Verbesserung $\mathrm{FEV}_{1}$ ) anders verhielten als diejenigen Kinder ohne positive 
Bronchospasmolyse. Tatsächlich zeigte es sich, dass Kinder mit gutem Ansprechen auf ein beta-2-Sympathomimetikum mehr reproduzierbare Ergebnisse im Rahmen der Exazerbation produzieren konnten als Kinder ohne positive Bronchospasmolyse. Dies ist unserer Meinung nach nicht ganz überraschend, da Kinder ohne signifikante Bronchospasmolyse im Rahmen einer infektbedingten Exazerbation häufiger mehr verschleimt sind und der Husten die Reproduzierbarkeit der Messung einschränkt. Allerdings stellt diese Beobachtung nur einen Trend dar, da keine Signifikanzen hierfür gefunden wurden. Ein weiterer Erklärungsansatz könnte die längere FET im Beschwerdeintervall sein. Man könnte annehmen, dass Kinder mit längerer FET aufgrund einer vorliegenden Obstruktion die forcierten exspiratorischen Manöver konsequenter bis zum Ende durchführen und die Obstruktion einen limitierenden Faktor für intraindividuelle Schwankungen darstellt. Warum letztlich im Rahmen der Exazerbation die Reproduzierbarkeit etwas besser war als im symptomfreien Intervall, bleibt aber unklar. Am ehesten ist davon auszugehen, dass im Rahmen der Verschlechterung aufgrund des Leidensdrucks die Konzentration und Motivation der Kinder höher war als im beschwerdefreien Intervall.

Zusammenfassend konnte unsere Arbeit zeigen, dass nach den neuen Kriterien der ATS von 2007 Kinder von $\geq 4$ bis $<7$ Jahren auch im exazerbierten Intervall akzeptable und reproduzierbare Lungenfunktionen leisten können. Die Arbeit stellt die erste Auswertung von Lungenfunktionsmessungen im exazerbierten Intervall nach neuen Kriterien in dieser Altersgruppe dar. Interessanterweise sind die Ergebnisse bei Beschwerden sogar besser als im gesunden Intervall. Auch konnte gezeigt werden, dass bis auf wenige Ausnahmen, insbesondere bei den 4-jährigen Patienten, der $\mathrm{FEV}_{1}$-Wert einen adäquaten Parameter zur Beurteilung einer vorliegenden Obstruktion darstellt. Bei FET-Zeiten von weniger als einer Sekunde sollten allerdings FEV ${ }_{0,75}$ bzw. FEV $\mathrm{F}_{0,5}$ herangezogen werden. Das 5\%-Reproduzierbarkeits-Kriterium, welches für vor allem für erwachsene Patienten in früheren Leitlinien festgelegt wurde, liefert im vorliegenden Patientenkollektiv signifikant weniger reproduzierbare Messungen und sollte zur Bewertung in dieser Altersgruppe nicht mehr herangezogen werden.

\section{Interessenkonflikt}

$\nabla$

Die Autoren geben an, dass kein Interessenkonflikt besteht.

\section{Literatur}

1 Brand PLP, Baraldi E, Bisgaard $H$ et al. Definition, assessment and treatment of wheezing disorders in preschool children: an evidence-based approach. Eur Respir J 2008; 32: 1096-1110

2 Martinez FD, Wright AL, Taussig LM et al. Asthma and wheezing in the first six years of life. The Group Health Medical Associates. N Engl J Med 1995; 332: $133-138$

3 Beydon N, Davis SD, Lombardi E et al. An official American Thoracic Society/European Respiratory Society statement: pulmonary function testing in preschool children. Am J Respir Crit Care Med 2007; 175: $1304-1345$

4 Nystad W, Samuelsen SO, Nafstad P et al. Feasibility of measuring lung function in preschool children. Thorax 2002; 57: 1021 - 1027

5 Nève V, Edmé J, Devos P et al. Spirometry in 3-5-year-old children with asthma. Pediatr Pulmonol 2006; 41: 735 - 743

6 Arets HG, Brackel HJ, van der Ent CK. Forced expiratory manoeuvres in children: do they meet ATS and ERS criteria for spirometry? Eur Respir J 2001; 18: $655-660$

7 Vilozni D, Barak A, Efrati $O$ et al. The role of computer games in measuring spirometry in healthy and „asthmatic“ preschool children. Chest 2005; 128: $1146-1155$

8 Loeb JS, Blower WC, Feldstein JF et al. Acceptability and repeatability of spirometry in children using updated ATS/ERS criteria. Pediatr Pulmonol 2008; 43: 1020-1024

9 Enright PL, Linn WS, Avol EL et al. Quality of spirometry test performance in children and adolescents: experience in a large field study. Chest 2000; 118: 665-671

10 Miller MR, Hankinson J, Brusasco Vet al. Standardisation of spirometry. Eur Respir J 2005; 26: 319- 338

11 American Thoracic Society. Standardization of Spirometry, 1994 Update. Am J Respir Crit Care Med 1995; 152: 1107-1136

12 Zielen S, Christmann M, Kloska $M$ et al. Predicting short term response to anti-inflammatory therapy in young children with asthma. Curr Med Res Opin 2010; 26: 483-492

13 Quanjer PH, Tammeling GJ, Cotes JE et al. Lung volumes and forced ventilatory flows. Work Group on Standardization of Respiratory Function Tests. European Community for Coal and Steel. Official position of the European Respiratory Society]. Rev Mal Respir 1994; 11 Suppl 3: 5-40

14 Aurora P, Stocks J, Oliver C et al. Quality control for spirometry in preschool children with and without lung disease. Am J Respir Crit Care Med 2004; 169: 1152 - 1159

15 Crenesse D, Berlioz M, Bourrier T et al. Spirometry in children aged 3 to 5 years: reliability of forced expiratory maneuvers. Pediatr Pulmonol 2001; 32: 56-61

16 Chavasse R, Johnson P, Francis J et al. To clip or not to clip? Noseclips for spirometry. Eur Respir J 2003; 21: 876-878

17 Gracchi $V$, Boel M, van der Laag J et al. Spirometry in young children: should computer-animation programs be used during testing? Eur Respir J 2003; 21: $872-875$ 